\title{
The Challenges of Embedded Systems Engineering
}

\author{
Ed Brinksma \\ Embedded Systems Institute, Eindhoven, The Netherlands
}

\begin{abstract}
Embedded system technology has become an important, if not dominating component in the realization of all sorts of high-tech products, machines, and infrastructures. The temptation to create systems with new, powerful, intelligent features has turned embedded software into an essential high-tech ingredient that, exploiting the hardware capabilities afforded by Moore's law, is subject to exponential growth. As has been pointed out by many authors before, the complexity of the embedded software is not just a product of its growing size, but also results from the required relation between the software and its physical environment, both in terms of its execution on physical platforms and in its interaction with the system environment. This combination of digital control and physical phenomena makes it plausible that hybrid modelling and hybrid systems theory have a role to play in the design of embedded systems. Last year Henzinger and Sifakis (The Embedded Systems Design Challenge) suggested that we need the development of more physically informed models of computation combining analytical and constructive elements. This would provide a more fundamental basis for embedded systems design, as well as provide a much needed paradigmatic change for computer science.

Being in principle sympathetic to the proposed programme from a scientific point of view, in this talk we want to examine what are the most pressing problems from an engineering point of view, in particular from the overall system perspective. The software complexity of hightech systems is often related to the system integration, and not to the embedded software of individual components. The interpretation of the required integral functionality usually includes engineering disciplines beyond those related to hardware, software, and control, with particular methods, models, and tools. It remains to be seen whether deep (i.e. at the semantic level) integration of relevant models and methods will ultimately outperform more loosely coupled coalitions of specialized approaches that are closer to the cultures of the contributing disciplines. Another practically dominant concern often is the sheer size of the collective system software. Alternative approaches to design, therefore, must scale up and support the management of large quantities of design software (programs, models, specifications, etc.). In our presentation we will draw on a number of big industry-as-laboratory projects carried out by the Embedded Systems Institute.
\end{abstract}

